This information is current as of April 26, 2023.

\title{
A Safe and Efficacious Alternative: Sonographically Guided Internal Jugular Vein Puncture for Intracranial Endovascular Intervention
}

C.-H. Yeh, Y.-M. Wu, C.-H. Toh, Y.-L. Chen and H.-F. Wong

AJNR Am J Neuroradiol 2012, 33 (1) E7-E12

doi: https://doi.org/10.3174/ajnr.A2416

http://www.ajnr.org/content/33/1/E7 
TECHNICAL NOTE

\author{
C.-H. Yeh \\ Y.-M. Wu \\ C.-H. Toh \\ Y.-L. Chen \\ H.-F. Wong
}

\section{A Safe and Efficacious Alternative: Sonographically Guided Internal Jugular Vein Puncture for Intracranial Endovascular Intervention}

\begin{abstract}
SUMMARY: Transvenous interventions for intracranial vascular lesions are usually performed via venous access of a femoral vein puncture. However, the transjugular route is an alternative with a shorter and less tortuous vascular access for intracranial lesions. Although puncture of the internal jugular vein is generally believed to be too dangerous owing to potential hazardous complications, the safety of the sonographically guided retrograde internal jugular vein puncture technique for intracranial intervention has not been fully evaluated in the English literature. We present our experience with a total of 44 transjugular intervention procedures between April 1999 and June 2010. We believe sonographically guided internal jugular vein puncture is a safe and efficacious technique for establishing transvenous access for an intracranial endovascular intervention.
\end{abstract}

ABBREVIATIONS: CCF = carotid cavernous fistula; DAVF = dural arteriovenous fistula; $\mathrm{ECA}=$ external carotid artery; GDC = Guglielmi detachable coil; ICA = internal carotid artery; $n$-BCA = $n$-butyl cyanoacrylate; SVC = superior vena cava; VA = vertebral artery
$T^{2}$ ransarterial access through arteriotomy of the femoral artery is most commonly used in intracranial endovascular neurointerventions. ${ }^{1}$ Through this transarterial route, endovascular procedures are performed for embolization of intracranial vascular lesions and hypervascular tumors. In addition, angioplasty and stent placement for arterial stenosis are also performed in a similar manner. However, some intracranial vascular lesions, including CCF, DAVF, or dural sinus thrombosis, are sometimes preferably managed by a transvenous access. $^{2,3}$ The transvenous routes can be established via common femoral vein puncture, internal jugular vein puncture, or direct puncture of abnormally dilated venous sacs. ${ }^{4}$ However, puncture of the internal jugular vein for intracranial intervention is generally believed to be dangerous due to the large sheaths used during the procedure, the difficulties in holding adequate pressure against the airway and other soft tissues of the neck during hemostasis, the potential serious complications of expanding cervical hematomas, and the risk of inadvertent carotid artery injury.

In our department, we routinely perform a sonographically guided technique in difficult cases of arterial and venous puncture. However, to our knowledge, the safety, efficacy, and potential risk of sonographically guided retrograde internal jugular vein puncture for intracranial intervention has not been fully evaluated in the English literature. In this study, we retrospectively reviewed the consecutive patients who underwent sonographically guided internal jugular vein puncture for an intracranial interventional procedure in our institution during the past 10 years. The safety, efficacy, and potential

Received July 12, 2010; accepted after revision September 14

From the Department of Medical Imaging and Intervention, Chang Gung Memorial Hospital, Linkou, and College of Medicine, Chang Gung University, Taoyuan, Taiwan.

Please address correspondence to Ho-Fai Wong. MD, Department of Medical College of Medicine, Imaging and Intervention, Chang Gung Memorial Hospital, Linkou, No. 5, Fu Hsing St, Gueishan, Taoyuan 333, Taiwan, R.O.C.; e-mail: hfwong@adm.cgmh.org. or hfwong@ms4.hinet.net

http://dx.doi.org/10.3174/ajnr.A2416 complications of this procedure were collected and analyzed in this study.

\section{Technique}

\section{Patients}

From April 1999 to June 2010, 44 transjugular intracranial interventions using a sonographically guided internal jugular vein puncture technique were performed in 42 patients at our institution. Two of these patients underwent a second transjugular intervention 2 months and 1.5 years, respectively, after the first procedure. All procedures were performed in accordance with the guidelines of the institutional review board, which approved our retrospective medical chart review and analysis.

\section{Procedure}

Except for 2 procedures of venous sampling, all procedures were performed with the patients under general anesthesia. The site of internal jugular vein puncture was chosen according to the site, location, and venous drainage pattern of the vascular lesion. Patency of the selected

Table 1: Patient demographics, coagulation profile, and diagnosis ${ }^{a}$

\begin{tabular}{lc}
\hline Demographics & Data \\
\hline Mean age (yr) & $52.5 \pm 17.3$ \\
Sex & 16 \\
$\quad$ Male & 26 \\
$\quad$ Female & \\
Coagulation profile & $11.09 \pm 0.09$ \\
$\quad$ Prothrombin time (international normalized ratio) & $25.74 \pm 3.33$ \\
Activated partial thromboplastin time & $223589 \pm 60921$ \\
Platelets & \\
Diagnosis & 11 \\
DAVF, without venous occlusion & 5 \\
DAVF, with venous occlusion & 1 \\
Venous occlusion & 22 \\
CCF & 1 \\
DAVF and CCF & 2 \\
$\quad$ Cushing syndrome & \\
\hline a $N=42$ &
\end{tabular}




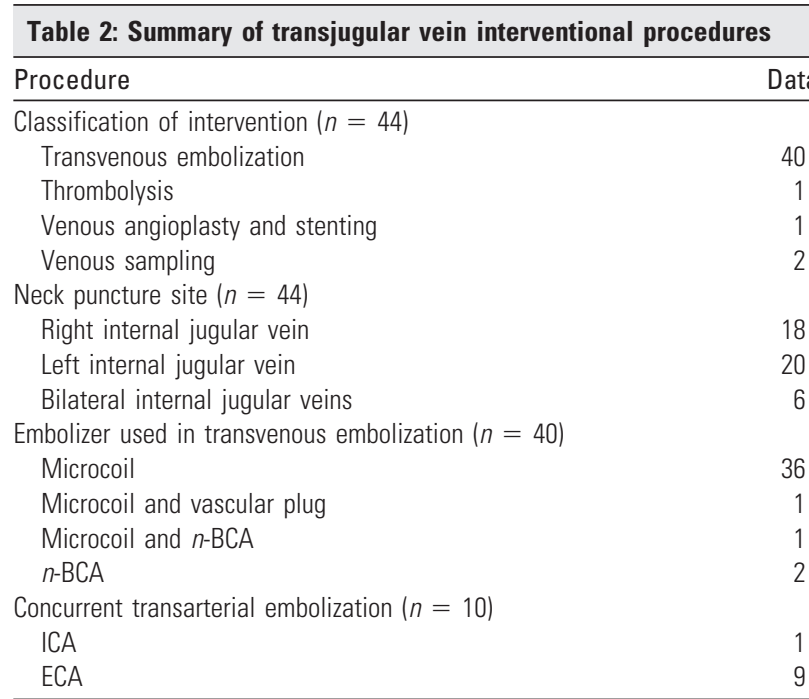

internal jugular vein was confirmed by using real-time sonography with compression and a color Doppler technique. A freehand technique was used in all patients. The selected internal jugular vein was punctured with an 18-ga needle catheter under sonographic guidance. A $6 \mathrm{~F}$ sheath was introduced into the internal jugular vein by using the Seldinger technique. Right common femoral artery puncture was performed with a conventional method. A $5 \mathrm{~F}$ or $5.5 \mathrm{~F}$ catheter was navigated into the feeding artery to obtain the venous road- map. If needed, complete angiography of bilateral ICAs, ECAs, and VAs was performed to assess the size and location and relationship of feeding arteries and drainage veins of the vascular lesion.

Selective catheterization of the dural venous sinus, usually the inferior petrosal sinus or the sigmoid sinus, was performed with a hydrophilic polymer-coated 0.035 -inch angled soft guidewire and a $5 \mathrm{~F}$ or $5.5 \mathrm{~F}$ end-hole guiding catheter. A microcatheter with a microwire was navigated coaxially to the optimal location for intervention. Then embolization or other procedures were performed with realtime digital subtraction fluoroscopic mapping. Angiography was performed immediately after completion of the procedure to check the outcome. Patents were generally followed by clinical evaluation. Conventional angiography or MR angiography re-evaluation was performed if clinically indicated.

\section{Results}

\section{Patient Population, Coagulation Profile, and Diagnosis}

The patient demographics, average of the coagulation profile, and indication for treatment are presented in Table 1. Coagulopathy (prolonged prothrombin time, activated partial thromboplastin time, or decreased platelet count) was found in only 1 patient. Among the 42 patients enrolled, 16 underwent transvenous embolization treatment for DAVFs. Cerebral venous occlusion was present in 5 and absent in 11 of these 16 patients with DAVFs. One patient had a superior sagittal sinus occlusion without a coexistent DAVF. Trans-
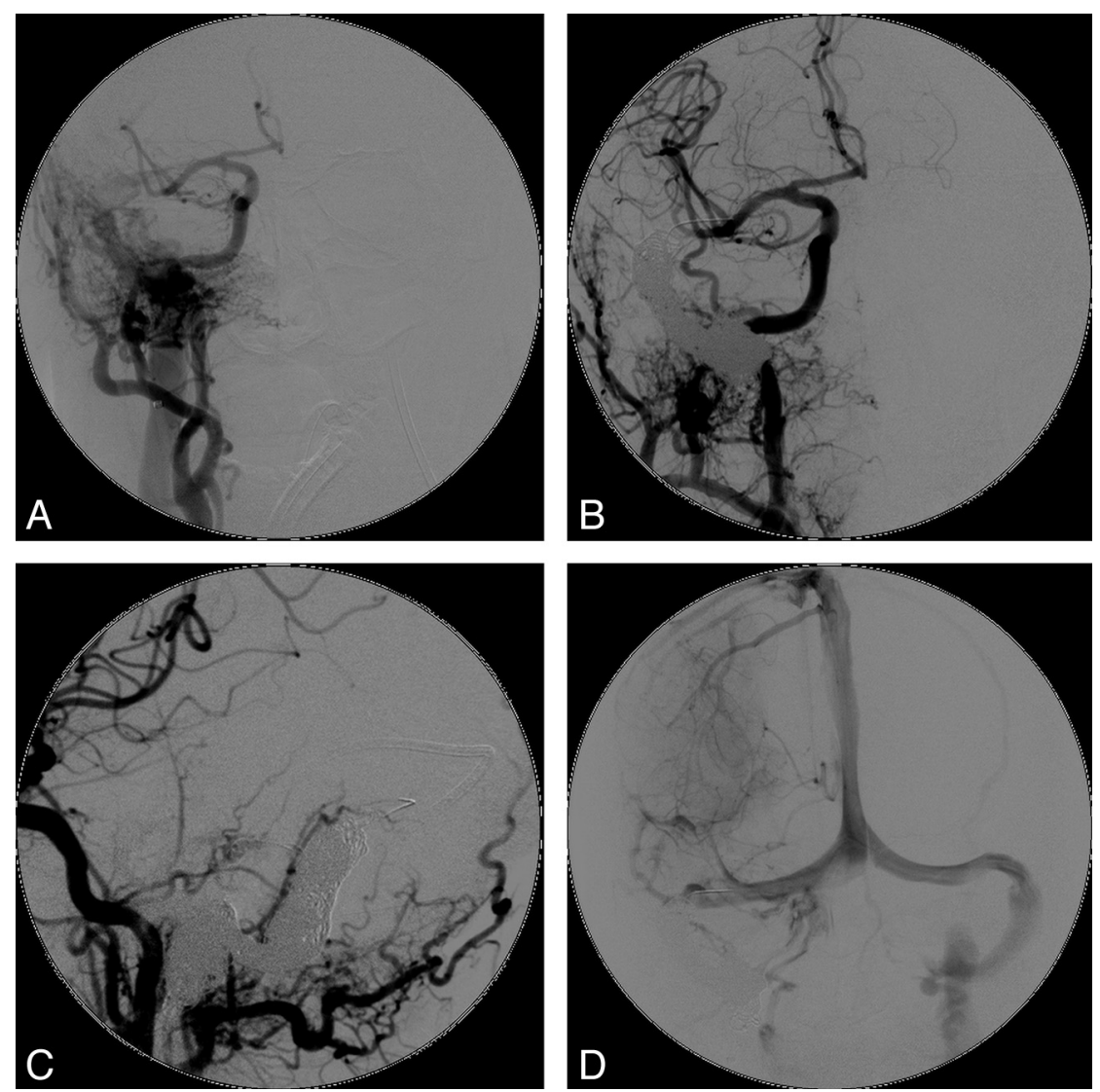

Fig 1. A 61-year-old woman with a right sigmoid sinus DAVF. A, Right internal carotid artery angiogram in an anteroposterior projection shows early opacification of the right sigmoid sinus and internal jugular vein with retrograde venous drainage through cerebral vein. $B$ and $C$, After venous sheaths are placed in the bilateral internal jugular veins, trapping of the right sigmoid sinus is performed with a vascular plug (through the right internal jugular vein) and $28 \mathrm{GDCs}$ (through the left internal jugular vein access). $D$, The fistula is completely obliterated after embolization. 

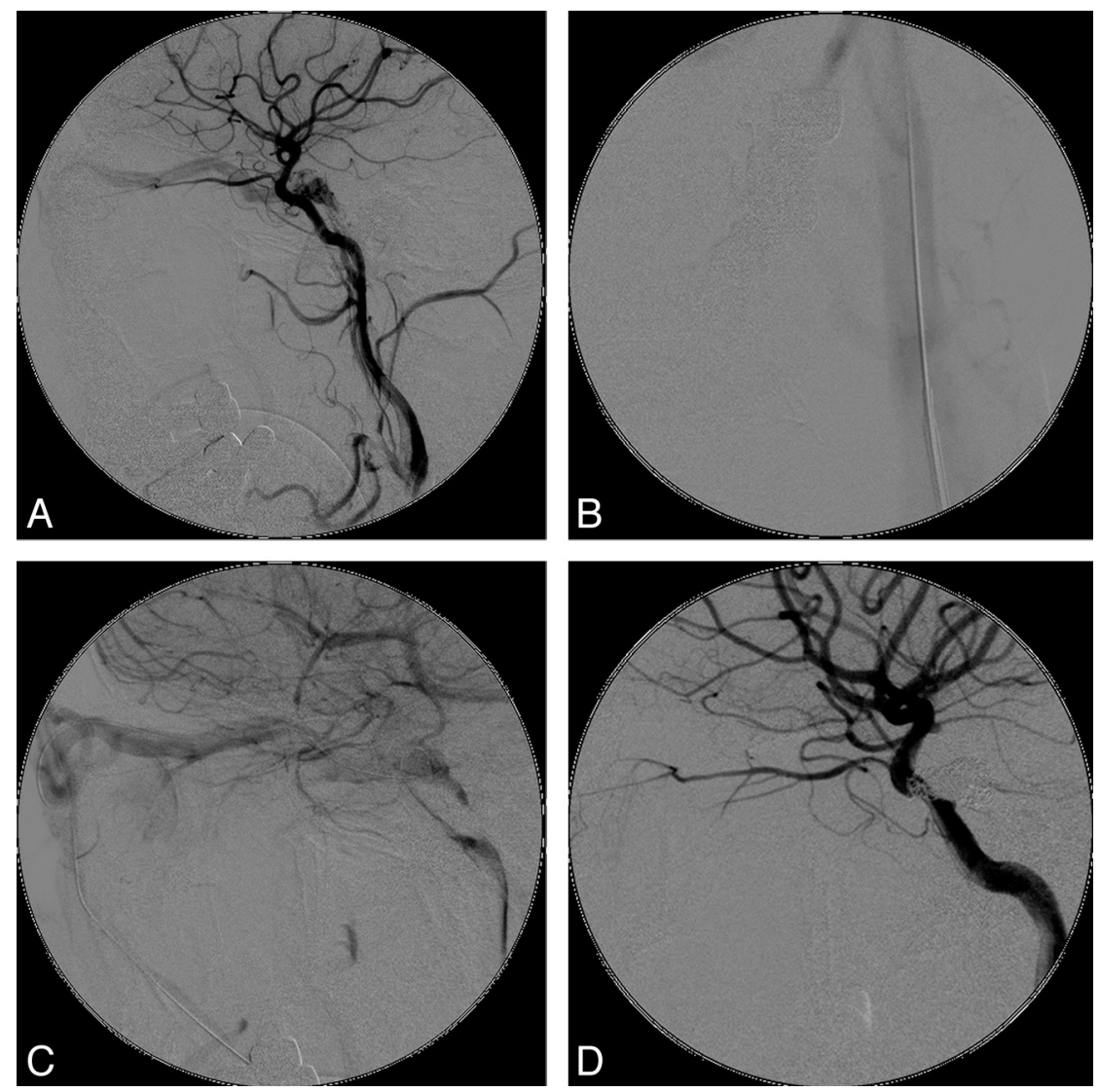

Fig 2. A 63-year-old man with a left indirect CCF. $A$, Left ICA angiogram in a lateral projection shows early opacification of the cavernous sinus with venous drainage mainly through the left superior ophthalmic vein. $B$ and $C$, After a $6 F$ sheath is placed into the left internal jugular vein $(B)$, a microcatheter is navigated through the left facial vein, left angular vein, and left superior ophthalmic vein into the left cavernous sinus $(C)$. After $16 \mathrm{GDCs}$ were deployed, the left cavernous sinus was completely occluded without residual fistula.

venous embolization of CCFs was performed in 23 patients. One patient with a coexistent CCF and a left jugular bulb DAVF had transvenous embolization for the CCF. Two patients with Cushing syndrome had left internal jugular puncture and right femoral vein puncture simultaneously for venous sampling.

\section{Sonographically Guided Internal Jugular Vein Puncture}

A sonographically guided puncture technique was performed in all 44 procedures. The internal jugular vein sheath was removed immediately after the procedure. After manual compression for 10 minutes, the venotomy wound was simply covered with gauze. No life-threatening complication or inadvertent carotid artery injury occurred. No additional procedure was required for hemostasis of the venotomy wound. No clinically discernible neck hematoma was found.

\section{Technique of Transjugular Vein Interventions}

Details of internal jugular vein puncture, transvenous intervention, and concurrent transarterial intervention are summarized in Table 2. Bilateral internal jugular vein puncture was performed in 6 patients to achieve optimal embolization therapy. In 2 of these 6 patients, a bilateral internal jugular vein approach was necessary for both proximal and distal segment embolization when trapping occluded dural sinuses and associated DAVFs (Fig 1). In the other 4 patients, puncture of the contralateral internal jugular was performed for alternative vascular access after failure of the initially planned trans- venous route. Catheterization was performed through the inferior petrosal sinus to the cavernous sinus in 23 patients; through the facial vein, angular vein, and superior ophthalmic vein to the cavernous sinus in 6 patients; to the superior petrosal sinus in 1 patient; through the dural venous sinuses to the superior sagittal sinus in 3 patients; and to the unilateral or contralateral jugular bulb, sigmoid, or transvenous or marginal sinuses in 11 patients. Transvenous embolization performed mainly with electrically detachable coils (GDCs; Boston Scientific, Natick, Massachusetts). One patient with superior sagittal sinus occlusion had mechanical and chemical thrombolysis. Jugular bulb angioplasty and stent placement were performed in 1 patient with jugular bulb occlusion and a DAVF. Concomitant transarterial embolization of feeding arteries with Onyx (ev3, Irvine, California), $n$-BCA, or particles was performed in 9 procedures.

\section{Procedure-Related Complications}

In 1 patient with a right marginal sinus DAVF, herniation and fixation of a microcoil into the internal jugular vein occurred. However, the internal jugular vein remained patent in subsequent follow-up angiography. In 1 patient with left transverse and sigmoid sinus DAVFs, embolization of the left superior petrosal sinus and engorged venous sacs in the left cerebropontine angle was performed to obliterate the extensive fistula and venous drainage. Unfortunately, left temporal intraparenchymal hemorrhage, subdural hemorrhage, and subarachnoid hemorrhage occurred immediately after embolization. This 

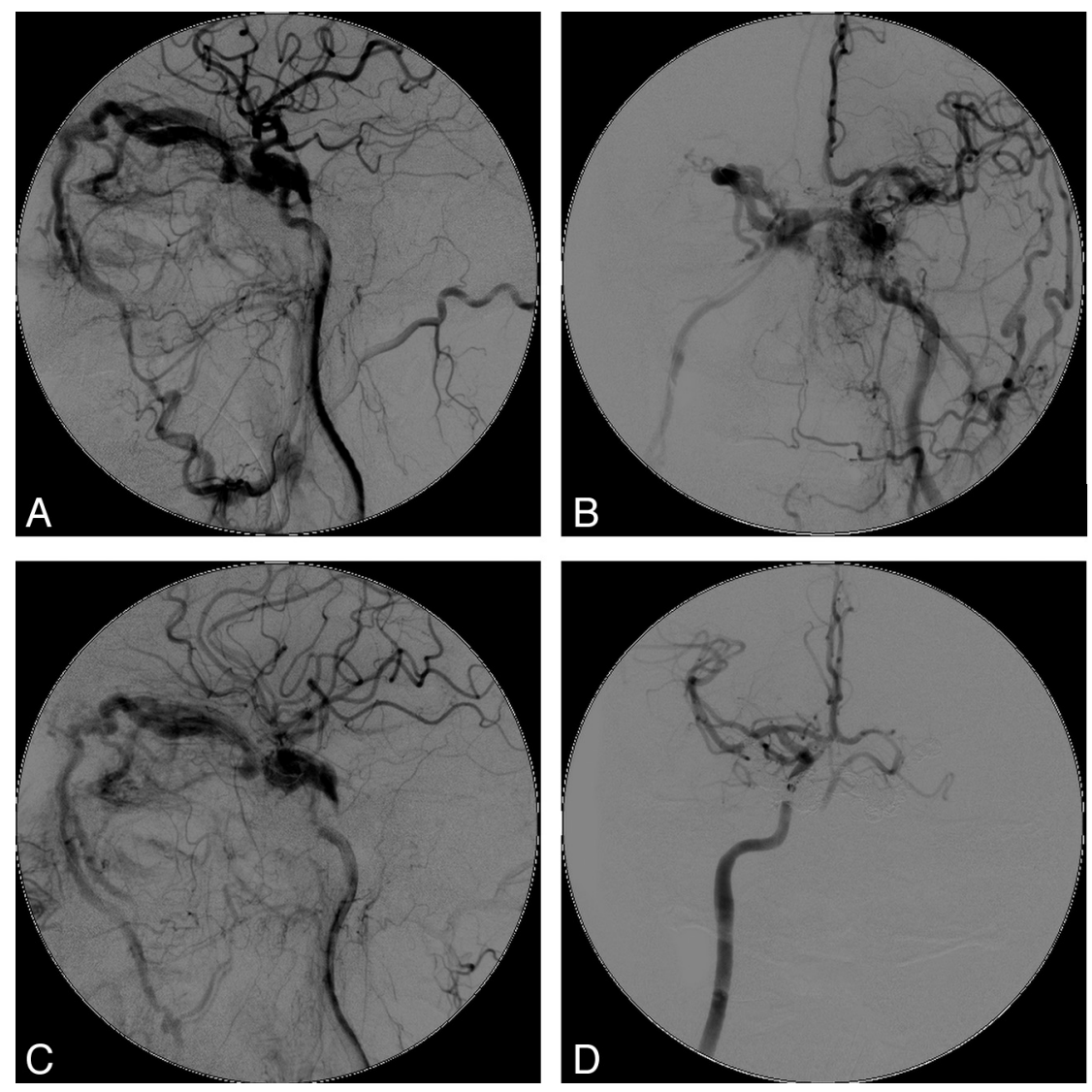

Fig 3. A 53-year-old man with bilateral indirect CCFs. $A$ and $B$, Left ICA angiogram in lateral $(A)$ and anteroposterior $(B)$ projections shows early opacification of bilateral cavernous sinuses with venous drainage through the bilateral superior ophthalmic vein. $C$, After the venous access is established, a microcatheter is navigated through the right facial vein, right superior ophthalmic vein, right cavernous sinus, and left cavernous sinus and into the left superior ophthalmic vein. Then embolization of bilateral superior ophthalmic veins and cavernous sinuses is performed with 31 GDCs. D, No residual fistula is seen on the postembolization angiogram.

patient died due to extensive intracranial hemorrhage. In a patient with bilateral CCFs, transarterial embolization of a right-sided fistula with Onyx was performed after optimal transvenous embolization of a left-sided fistula with microcoils. However, regurgitation of Onyx though the residual fistula into the left ICA occurred. The cavernous and clinoid segments of left ICA were completely occluded. Patent collateral perfusion through the anterior communicating artery and left posterior communicating artery was confirmed immediately on right ICA angiography. The patient had only a minor infarct in the left corona radiata and body of the left corpus callosum and recovered smoothly without major neurologic deficits. However, none of these patients had morbidity or mortality associated with the internal jugular vein puncture.

\section{Discussion}

Transvenous endovascular intervention is a well-established technique for many intracranial vascular diseases. Through the venous route, the interventional radiologist can perform embolization of CCFs and DAVFs. ${ }^{2,5-7}$ Thrombolysis, embolectomy, and stent placement for dural sinus thrombosis can also be achieved transvenously. ${ }^{3,8,9}$ In addition, venous sampling is also performed transvenously for diagnosis of pituitary gland disorders. ${ }^{10,11}$ Although femoral vein puncture is the most commonly used venous access, jugular vein puncture and direct puncture of engorged veins in the head and neck are possible alternatives. ${ }^{5,7}$
Currently, the internal jugular vein puncture technique is widely used for placement of central venous catheters, tunneled central catheters for hemodialysis, ${ }^{12-14}$ and inferior vena cava filters. ${ }^{15,16}$ Ablation of atrial fibrillation, ${ }^{17}$ transjugular intrahepatic portosystemic shunt, ${ }^{18}$ and transvenous hepatic biopsy ${ }^{19,20}$ are also reported to be safe via transjugular access. The interventionist can benefit from the internal jugular puncture in many aspects, compared with using the common femoral vein puncture. First, the internal jugular vein is much closer to the intravascular vascular lesion. With the shorter route of catheterization, a shorter guiding catheter can be used. As a result, the available working length of the microcatheter and microwire would be much longer for selective catheterization during the procedure. In addition, with the shorter catheter retained in the patient's body, the risk of iatrogenic thromboembolism would be less during the procedure. Second, several angulations of the guiding catheter are often encountered at the junctions between the SVC and brachiocephalic veins, between the brachiocephalic veins and internal jugular veins, and at the jugular bulbs in a transfemoral route technique. With the transjugular route, the 2 marked angulations at the junctions between the SVC and brachiocephalic veins and between the brachiocephalic veins and internal jugular vein are bypassed. Therefore, less tortuosity of the catheter and, correspondingly, less difficulty of catheterization and embolization would be encountered during the procedure. 

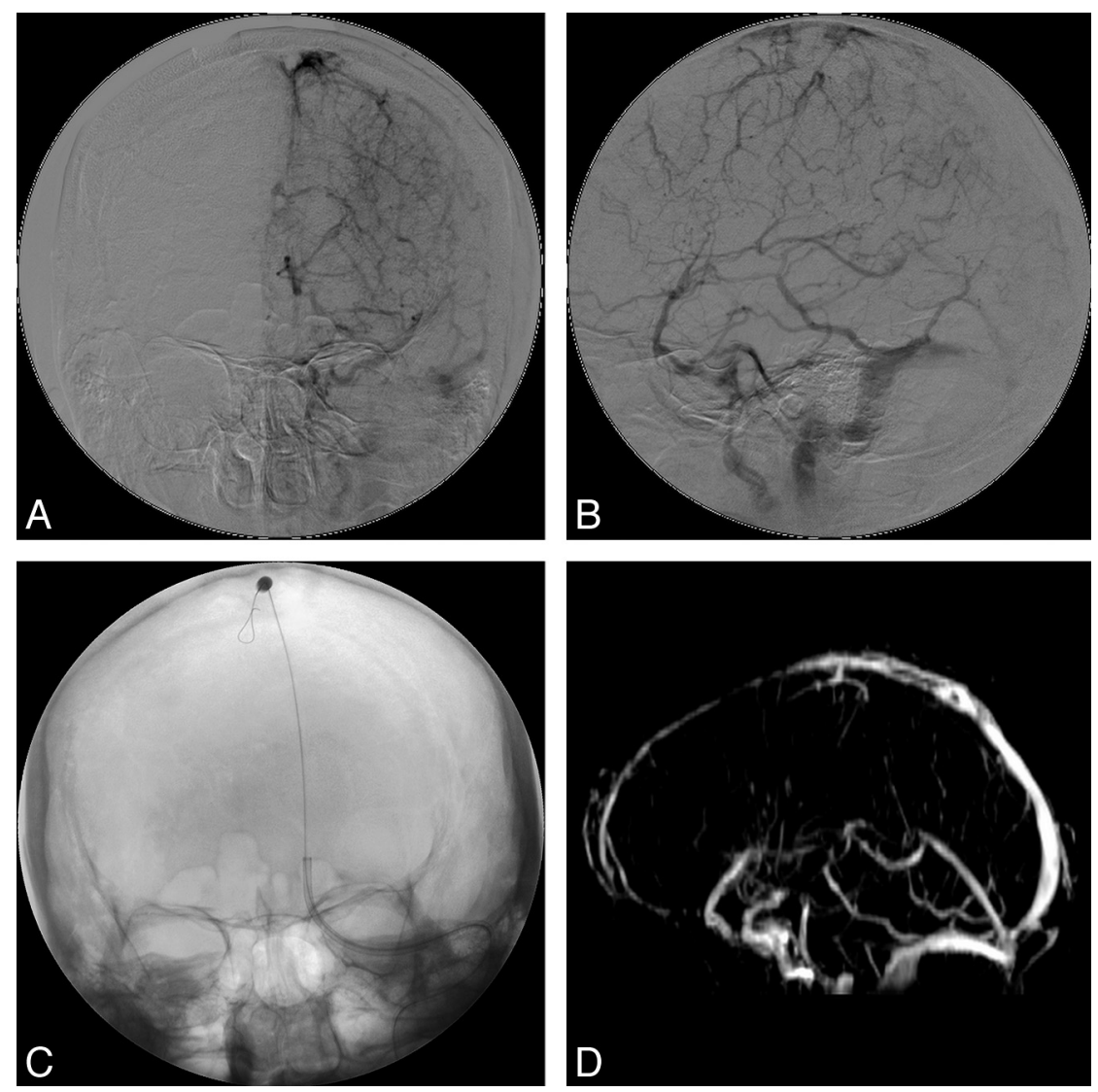

Fig 4. A 54-year-old woman with superior sagittal sinus thrombosis. $A$ and $B$, Left internal carotid artery angiogram, venous phase, in anteroposterior $(A)$ and lateral $(B)$ projections shows occlusion of superior sagittal sinus, right transverse sinus, right sigmoid sinus, and left transverse sinus (not shown). $C$, After a $6 \mathrm{~F}$ guiding catheter is placed into the superior sagittal sinus, angioplasty with a balloon catheter is performed. $D$, After subsequent 48-hour chemical thrombolysis with urokinase, recanalization of the superior sagittal sinus and left transvenous sinus is confirmed in MR venography 3 days and 6 months later (not shown).

In the case presented in Figs 2 and 3, the microcatheter was successfully navigated through the facial vein, angular vein, and superior ophthalmic vein into the cavernous sinus and inferior petrosal sinus. With the benefit of a transjugular vein approach, the tortuosity of the guiding catheter and microcatheter was markedly decreased. Correspondingly, the resistance during advancing the catheter and deploying microcoils was also reduced. Therefore, the procedure can be performed more smoothly with less operation time. Furthermore, the 1 -way valves at the junction of internal jugular vein and brachiocephalic vein would impede retrograde catheterization when performing a transfemoral technique, but they are easily bypassed by transjugular routes.

Sometimes, endovascular treatments are performed via sonographically guided puncture of pathologically dilated venous sacs or the drainage vein near the vascular lesion. Theoretically, the route of catheterization would be shortest by these vascular accesses. However, due to the relatively smaller caliber and tortuous nature of these venous routes, it can be difficult to place a large-sized vascular sheath, such as the $6 \mathrm{~F}$ sheath we used in the transjugular vein technique. With the small-sized vascular sheath, the available guiding catheter, guidewire, microcatheter, microwire, and special technique of catheterization (eg, the double-catheter technique and balloon-assisted embolization) are all limited during the procedure. As in the case presented in Fig 4, recannulation of the occluded superior sagittal sinus was performed through left internal jugular vein access. With the benefit of a large-sized (6F) internal jugular vein sheath, a balloon catheter was deployed and inflated without difficulty. In addition, no limitation of trunk or limb movement was required during the subsequent 48 -hour chemical thrombolysis. Finally, patients with obesity are not uncommon in the daily practice of interventional neuroradiologists. The marked thick layer of subcutaneous fatty tissue and the deep skin folds around the groin area can make femoral vein puncture time-consuming and sometimes impossible. In these patients, internal jugular vein puncture is another alternative technique.

There are also some drawbacks to the internal jugular vein puncture for intracranial interventions. First, internal jugular vein punctures are usually performed antegradely in other procedures (eg, in transjugular hepatic interventions). However, they are uniquely performed retrogradely during intracranial interventions. When one performs the retrograde jugular vein puncture, the conventional landmark guidance technique used in antegrade puncture is not useful. Moreover, retrogradely navigating a guidewire and catheter within a transvenous route would be markedly difficult due to the reversed direction of blood flow and the complex network of venous return in the head and neck regions. Therefore, we routinely use the technique of indirectly obtaining a venous roadmap with a transarterial catheter in the feeding artery to overcome these difficulties.

The congenital variation of the head and neck venous net- 
work also increases the difficulty of the transjugular technique. For example, the confluence of the facial vein or occipital vein with the internal jugular vein may be located in the middle or lower portion of the neck. They will be bypassed after placement of a vascular sheath with the puncture site in the middle neck according to the conventional landmark guidance technique. Therefore, it is wise to make the site of the internal jugular vein puncture as caudally as possible. As in other transjugular interventions, we are always concerned about the potential inadvertent injury of the carotid artery and venous bleeding due to repeat puncture or penetration of the posterior wall of the jugular vein in patients with coagulopathy. ${ }^{21}$ Under ultrasonographic guidance, internal jugular vein puncture can be performed safely and accurately at the optimal venous access site. Therefore, the risk of carotid artery injury, uncontrollable neck bleeding, and neck hematoma are markedly decreased. In this study, transjugular accesses were successfully established in all 44 procedures with sonographic guidance without inadvertent carotid artery injury. No puncture-related morbidity or mortality occurred during any of these procedures.

\section{Conclusions}

Sonographic guidance of internal jugular vein puncture is a useful procedure in establishing a transvenous route for an intracranial endovascular intervention. According to our 10year experience of intracranial intervention, transvenous accesses were successfully established with sonographic guidance in 44 procedures. No morbidity or mortality associated with the internal jugular vein puncture occurred. Therefore, we believe this technique be a safe and efficacious alternative vascular access for intracranial endovascular interventions.

\section{References}

1. Kiyosue H, Hori Y, Okahara M, et al. Treatment of intracranial dural arteriovenous fistulas: current strategies based on location and hemodynamics, and alternative techniques of transcatheter embolization. Radiographics 2004;24:1637-53

2. Klisch J, Huppertz HJ, Spetzger U, et al. Transvenous treatment of carotid cavernous and dural arteriovenous fistulae: results for 31 patients and review of the literature. Neurosurgery 2003;53:836-56, discussion 56-57
3. Rahman M, Velat GJ, Hoh BL, et al. Direct thrombolysis for cerebral venous sinus thrombosis. Neurosurg Focus 2009;27:E7

4. Yoshida K, Melake M, Oishi $\mathrm{H}$, et al. Transvenous embolization of dural carotid cavernous fistulas: a series of 44 consecutive patients. AJNR Am J Neuroradiol 2010;31:651-55

5. Ng PP, Higashida RT, Cullen S, et al. Endovascular strategies for carotid cavernous and intracerebral dural arteriovenous fistulas. Neurosurg Focus 2003; 15:ECP1

6. Biondi A, Milea D, Cognard C, et al. Cavernous sinus dural fistulae treated by transvenous approach through the facial vein: report of seven cases and review of the literature. AJNR Am J Neuroradiol 2003;24:1240-46

7. Berkmen T, Troffkin NA, Wakhloo AK. Transvenous sonographically guided percutaneous access for treatment of an indirect carotid cavernous fistula. AJNR Am J Neuroradiol 2003;24:1548-51

8. Masuhr F, Einhaupl K. Treatment of cerebral venous and sinus thrombosis. Front Neurol Neurosci 2008;23:132-43

9. Canhao P, Falcao F, Ferro JM. Thrombolytics for cerebral sinus thrombosis: a systematic review. Cerebrovasc Dis 2003;15:159-66

10. Boscaro M, Arnaldi G. Approach to the patient with possible Cushing's syndrome. J Clin Endocrinol Metab 2009;94:3121-31

11. Tomycz ND, Horowitz MB. Inferior petrosal sinus sampling in the diagnosis of sellar neuropathology. Neurosurg Clin N Am 2009;20:361-67

12. Koroglu M, Demir M, Koroglu BK, et al. Percutaneous placement of central venous catheters: comparing the anatomical landmark method with the radiologically guided technique for central venous catheterization through the internal jugular vein in emergent hemodialysis patients. Acta Radiol 2006;47:43-47

13. Docktor BL, Sadler DJ, Gray RR, et al. Radiologic placement of tunneled central catheters: rates of success and of immediate complications in a large series. AJR Am J Roentgenol 1999;173:457-60

14. Pittiruti M, Malerba M, Carriero C, et al. Which is the easiest and safest technique for central venous access? A retrospective survey of more than $\mathbf{5 4 0 0}$ cases. J Vasc Access 2000;1:100-07

15. Aziz F, Spate K, Wong J, et al. Changing patterns in the use of inferior vena cava filters: review of a single center experience. J Am Coll Surg 2007;205:564-69

16. Kinney TB. Update on inferior vena cava filters. J Vasc Interv Radiol 2003;14:425-40

17. Lim HE, Pak HN, Tse HF, et al. Catheter ablation of atrial fibrillation via superior approach in patients with interruption of the inferior vena cava. Heart Rhythm 2009;6:174-79. Epub 2008 Nov 1

18. Lorenz JM. Placement of transjugular intrahepatic portosystemic shunts in children. Tech Vasc Interv Radiol 2008;11:235-40

19. Soyer P, Fargeaudou Y, Boudiaf M, et al. Transjugular liver biopsy using ultrasonographic guidance for jugular vein puncture and an automated device for hepatic tissue sampling: a retrospective analysis of 200 consecutive cases. $A b$ dom Imaging 2008;33:627-32

20. Smith TP, Presson TL, Heneghan MA, et al. Transjugular biopsy of the liver in pediatric and adult patients using an 18-gauge automated core biopsy needle: a retrospective review of 410 consecutive procedures. AJR Am J Roentgenol 2003;180:167-72

21. Blaivas M, Adhikari S. An unseen danger: frequency of posterior vessel wall penetration by needles during attempts to place internal jugular vein central catheters using ultrasound guidance. Crit Care Med 2009;37:2345-49, quiz 59 